COMMUNICATIONS IN

ANALYSIS AND GEOMETRY

Volume 13, Number 5, 963-979, 2005

\title{
Constant mean curvature foliations of simplicial flat spacetimes
}

\author{
LARS ANDERSSON ${ }^{1}$
}

\begin{abstract}
Benedetti and Guadagnini [5] have conjectured that the constant mean curvature foliation $M_{\tau}$ in a $2+1$ dimensional flat spacetime $V$ with compact hyperbolic Cauchy surfaces satisfies $\lim _{\tau \rightarrow-\infty} \ell_{M_{\tau}}=s_{\mathcal{T}}$, where $\ell_{M_{\tau}}$ and $s_{\mathcal{T}}$ denote the marked length spectrum of $M_{\tau}$ and the marked measure spectrum of the $\mathbb{R}$-tree $\mathcal{T}$, dual to the measured foliation corresponding to the translational part of the holonomy of $V$, respectively. We prove that this is the case for $n+1$ dimensional, $n \geq 2$, simplicial flat spacetimes with compact hyperbolic Cauchy surface. A simplicial spacetime is obtained from the Lorentz cone over a hyperbolic manifold by deformations corresponding to a simple measured foliation.
\end{abstract}

\section{Introduction.}

In this paper, we will consider maximal, globally hyperbolic, flat (MGHF) spacetimes $V$ of dimension $n+1, n \geq 2$, with compact Cauchy surface $M$ of hyperbolic type, i.e. which admits a metric $g$ of constant sectional curvature -1 . The main result of the present paper implies, in the $2+1$ dimensional case, the proof of a conjecture of Benedetti and Guadagnini [5, Conj. 5.1], see conjecture 1.1 below, in the special case of simplicial flat spacetimes. A simplicial spacetime is a flat spacetime which can be obtained from the Lorentz cone over $M$, with metric $-d \rho^{2}+\rho^{2} g_{0}$ over $M$, by performing certain deformations relating to a weighted, finite collection of non-intersecting, compact, simple (i.e. without self-intersections), totally geodesic hypersurfaces $\mathcal{L}=\left\{\left(\Sigma_{k}, \ell_{k}\right), k=1, \ldots, m\right\}$, with weights $\ell_{k} \in \mathbb{R}$, in $\left(M, g_{0}\right)$. In particular, a simplicial spacetime has a compact Cauchy surface of hyperbolic type. Let $I_{\ell}=[0, \ell]$. The deformation corresponding to a single such hypersurface $(\Sigma, \ell)$ corresponds to gluing in a "wedge" spacetime

\footnotetext{
${ }^{1}$ Supported in part by the Swedish Research Council, contract no. R-RA 4873307, the NSF, contract no. DMS 0104402, and the Erwin Schrödinger Institute, Vienna.
} 
$W_{\ell}=W \times I_{\ell}$, in place of the Lorentz cone $W$ over $\Sigma$. In case $n=2, \mathcal{L}$ is a "weighted multicurve", or simple measured foliation with compact, simple geodesic leaves (by [21], every totally geodesic hypersurface of a compact hyperbolic manifold of dimension $n \geq 3$ is compact). Further, if $n=2$, simple measured foliations with compact leaves are dense in the space of all measured foliations.

A MGHF spacetime $V$ with compact Cauchy surface $M$ of hyperbolic type is globally foliated by CMC hypersurfaces $M_{\tau}$ with $\tau$ taking all values in $(-\infty, 0)$, see [1]. Further, the scale free version $\frac{\tau^{2}}{n^{2}} g_{\tau}$ of the induced metric $g_{\tau}$ on $M_{\tau}$ converges in the expanding direction, as $\tau \nearrow 0$, to a metric of constant sectional curvature -1 . In case $n \geq 3$, this metric is the unique hyperbolic metric on $M$, while in case $n=2$, this metric corresponds to a point in the Teichmuller space Teich $(M)$ of $M$. This is a partial generalization of the results for the case $n=2$ proved in [4]. In that paper, it was also proved that in the direction $\tau \searrow-\infty$, towards the singularity, the Teichmuller class of the induced metric on $M_{\tau}$ diverges, in the sense that it leaves every compact subset of Teich $(M)$, as $\tau \searrow-\infty$. This is proved by showing that the Dirichlet energy $\mathcal{E}$, which is a proper function on Teich $(M)[18$, Section 3], see also [20], diverges as $\tau \searrow-\infty$. However, the work in [4] does not give a detailed picture of the geometry of the CMC hypersurfaces $M_{\tau}$ for $\tau \searrow-\infty$. It is the purpose of this paper to study the detailed asymptotic behavior of the geometry of $M_{\tau}$ in the case when $V$ is simplicial.

\subsection{Flat spacetimes, earthquakes and $\mathbb{R}$-trees.}

A time oriented MGHF spacetime $V$ with oriented Cauchy surface $M$ may be viewed as an $\mathrm{ISO}^{+}(n, 1)$ geometric structure, and as such is described by the holonomy representation $\alpha$ of the fundamental group $\pi_{1}(M)$ in $\mathrm{ISO}^{+}(n, 1)$. The decomposition $\operatorname{ISO}(n, 1)=\mathrm{SO}(n, 1) \ltimes \mathbb{R}^{n+1}$ leads to a decomposition $\alpha(\gamma)=Q(\gamma)+t_{\gamma}$ where $Q$ is the linear part of the holonomy and the translational part $t_{\gamma}$ is a cocycle with values in $\mathbb{R}^{n+1}$. The linear part $Q$ of $\alpha$ corresponds to a hyperbolic structure on $M$. Let $\Gamma=\alpha\left(\pi_{1}(M)\right) \subset$ $\mathrm{ISO}^{+}(n, 1)$. The moduli space of MGHF spacetimes with Cauchy surface $M$ is homeomorphic to the Zariski tangent space $H^{1}\left(\Gamma, \mathfrak{i s o}^{+}(n, 1)\right.$ Ad $)$, see $[13,1]$. We denote by $i \mathfrak{s o}$ and $\mathfrak{s o}$, the Lie algebras of ISO and SO, respectively, and Ad indicates that $\Gamma$ acts by the adjoint representation.

In case $n=2$, the dimension of this space is 12 genus $(M)-12$, twice the dimension of Teichmuller space, while for $n \geq 3$, by Mostow rigidity, we have $H^{1}\left(\Gamma, \mathfrak{i s o}^{+}(n, 1)_{\mathrm{Ad}}\right)=H^{1}\left(\Gamma, \mathbb{R}_{\text {vec }}^{n+1}\right)$. For $n=3$, the dimension of the moduli space of flat spacetimes is the same as that of the tangent space 
of the space of flat conformal structures, $H^{1}\left(\Gamma, \mathbb{R}_{\text {vec }}^{3+1}\right)=H^{1}\left(\Gamma, \mathfrak{s o}(4,1)_{\text {Ad }}\right)$ [12, Section 11]. In case $n=2$, the translational part $t$ corresponds to a measured foliation $\mathcal{F}$ of $M$. Given a measured foliation $\mathcal{F}$ of a hyperbolic surface $M$, there is a unique geometric real tree $(\mathbb{R}$-tree) $(\mathcal{T}, d)$ dual to it. The fundamental group $\pi_{1}(M)$ acts in a natural way on $\mathcal{T}$.

Returning to the case of general dimension $n$, the development $\mathcal{D}(\mathrm{U}(V))$ of the universal cover $\mathrm{U}(V)$ in $\mathbb{M}^{n+1}$, the $n+1$ dimensional Minkowski space, is a convex open subset, the boundary $\mathbf{H}$ of which is the Cauchy horizon of $\mathrm{U}(V)$. From general results in causality theory, $\mathbf{H}$ is a weakly spacelike $C^{0}$ hypersurface. The fundamental group $\pi_{1}(M)$ acts on $\mathcal{D}(\mathrm{U}(V))$ and the action extends to $\mathbf{H}$. The Lorentz structure of $\mathbb{M}^{n+1}$ induces a degenerate distance function $d$ on $\mathbf{H}$. In the simplicial case, if we identify points of $\mathbf{H}$ under the equivalence relation $\sim$ defined by $p \sim q$ if $d(p, q)=0$, the action of $\pi_{1}(M)$ drops to the quotient $\mathbf{H} / \sim$. The metric space $\mathbf{H} / \sim$ can be identified with $(\mathcal{T}, d)$, which in this case is simplicial.

The cosmological time function $\rho(p)$, see $[2]$, on $\mathcal{D}(\mathrm{U}(V)) \subset \mathbb{M}^{n+1}$, defined as the maximal Lorentz length of any past directed causal curve starting at $p$ in $\mathcal{D}(\mathrm{U}(V))$ is regular, i.e. it is everywhere finite and $\rho \rightarrow 0$ along every past directed inextendible causal curve. This construction drops to the quotient $V$. Starting from the work of Mess [13], Benedetti and Guadagnini [5] showed that in case $n=2$, the induced geometry of the level sets of the cosmological time function $\rho$ introduced in [2] realize the Thurston earthquake deformation, in the sense that the curve in Teichmuller space defined by the Teichmuller class of the induced geometry of the level sets $M_{\rho}$ of the cosmological time function corresponds to the Thurston earthquake flow, defined with respect to the hyperbolic structure given by $Q$ and the measured foliation $\mathcal{F}$, see [5, Prop. 4.27 and Section 4.6]. In particular, as $\rho \rightarrow \infty$, the Teichmuller class of $M_{\rho}$ converges to the hyperbolic surface $M$ with holonomy $Q$, while as $\rho \rightarrow 0$, the geometry of the universal cover $\mathrm{U}\left(M_{\rho}\right)$ converges in the Gromov sense to an $\mathbb{R}$-tree $\mathcal{T}$, determined by the translational part $t$ of the holonomy $\alpha$ of $V$.

The $\mathbb{R}$-tree $\mathcal{T}$ can be identified with a point on the Thurston boundary of Teichmuller space. To explain this fact, we need the notions of marked length spectrum and marked measure spectrum, which we introduce following [5, Section 4.5]. Let $(\tilde{X}, d)$ be a metric space with an action $\alpha$ of $\pi_{1}(M)$ and $X=\tilde{X} / \pi_{1}(M)$. Let $\mathcal{C}$ be the space of conjugation classes of $\pi_{1}(M) \backslash\{1\}$. Then, $\mathcal{C}$ can be identified with the space of non-trivial homotopy classes of closed curves on $M$. For $c \in \mathcal{C}$, the marked length spectrum $s_{X}(c)$ is defined as $s_{X}(c)=\inf _{p \in \tilde{X}} d(p, \alpha(p))$. In case $X$ is homeomorphic to $M$, this corresponds to the shortest length of closed curves in $c$, and 
is denoted by $\ell_{X}$. In particular, by letting $X$ vary among the hyperbolic structures on $M, s_{X}$ gives a map $s_{X}: \operatorname{Teich}(M) \rightarrow \mathbb{R}_{\geq 0}^{\mathcal{C}}$, which is strictly positive.

On the other hand, in case $\tilde{X}=\mathcal{T}, s_{\mathcal{T}}(c)$ can be expressed in terms of the measured foliation $\mathcal{F}$ of $M$ dual to $\mathcal{T}$ as the minimal transverse measure realized by the curves in $c$. This gives the marked measure spectrum $I_{\mathcal{F}}$. If $\mathcal{F}$ is a simple measured foliation $\mathcal{L}$ with compact leaves, then $I_{\mathcal{L}}(c)$ is defined in terms of the geometric intersection number of the curves in $c$ with $\mathcal{L}$.

This extends the notion of length spectrum to the degenerate case. In the 2 dimensional case, the image of Teich $(M)$ under $\ell_{X}$ is homeomorphic to the open ball in $\mathbb{R}^{6 \text { genus }(M)-6}$. The boundary consists of degenerate geometries corresponding to projective rays in the image of the space of measured foliations under $I$. This is the Thurston boundary of Teichmuller space. The convergence of marked spectra can be understood as convergence of metric spaces in the Gromov sense, see [5, Remark 4.24, point 3)].

By [5], the foliation $M_{\rho}$ gives an analytic curve in Teichmuller space connecting the interior point $(M, Q)$ to the point on the Thurston boundary corresponding to $\mathcal{T}$. Thus, the spacetime geometry allows us to recover all the information about the holonomy in a concrete way. In the particular case of a $2+1$ dimensional flat simplicial spacetime, defined by a hyperbolic surface $M$ and a simple measured lamination with compact leaves $\mathcal{L}$ on $M$, the Teichmuller class of the level sets $M_{\rho}$ of the cosmological time function sweep out a curve corresponding to the Fenchel-Nielsen deformation of $M$ obtained by twisting $M$ along the closed geodesics $\Sigma_{k}$ of $\mathcal{L}$, and the geometry on $\mathrm{U}\left(M_{\rho}\right)$ converges in the Gromov sense to the simplicial tree $\mathcal{T}$ dual to $\mathcal{L}$. We refer to $[13,5,14]$ for background on the concepts discussed above.

The conjecture of Benedetti and Guadagnini can now be stated as follows:

Conjecture 1.1 ([5, Conj. 5.1]). Let $V$ be a $2+1$ dimensional MGHF spacetime with compact Cauchy surface of genus $\geq 2$, and let $M_{\tau}$ be the foliation of $V$ by constant mean curvature hypersurfaces with mean curvature $\tau$. Then
1. $\lim _{\tau \rightarrow-\infty} \ell_{M_{\tau}}=s_{\mathcal{T}}$,
2. $\lim _{\tau \rightarrow 0} \ell_{M_{\tau}} / \tau=\ell_{M}$.

Point (2), which states that the scale-free geometry on $M_{\tau}$ converges to the hyperbolic geometry $(M, g)$ corresponding to the holonomy $Q$ in the expanding direction $\tau \rightarrow 0$, follows for $n \geq 2$ from the work in [1]. In this 
paper, we will prove that the statement corresponding to point (1) is true for simplicial MGHF spacetimes with compact Cauchy surface of hyperbolic type, of general dimension $n \geq 2$. We can state the main result of this paper as follows, see Theorem 3.1.

Theorem 1.2. Let $V$ be an $n+1$ dimensional simplicial spacetime and let $M_{\tau}$ be the foliation of $V$ by constant mean curvature hypersurfaces with mean curvature $\tau$. Then $\lim _{\tau \rightarrow-\infty} \ell_{M_{\tau}}=s_{\mathcal{T}}$.

Recall that in case $n=2$, the simple measured foliations with compact leaves are dense in the space of all measured foliations. It is therefore natural to conjecture that the result proved here will yield the general case by a limit argument. We will not consider this problem here.

Our results here hold for simplicial flat spacetimes in general dimension $n+1, n \geq 2$. The relation of the case of simplicial flat spacetimes to the general case can be expected to be quite complicated in higher dimensions. In fact, Scannell [16] showed there are non-rigid, compact, hyperbolic 3manifolds (i.e. ones with $\left.H^{1}\left(\Gamma, \mathfrak{s o}(4,1)_{\text {Ad }}\right) \neq\{0\}\right)$ which have no immersed totally geodesic hypersurfaces. Therefore, it is not clear if the $2+1$ dimensional picture described above generalizes to the higher dimensional case. It is an interesting open problem to describe the asymptotics of both the foliation by level sets of the cosmological time function and of the CMC foliation of general higher dimensional flat spacetimes.

One of the main ideas in the work of Benedetti and Guadagnini is that the foliation by level sets of the cosmological time function realizes in a natural way the earthquake deformation of Thurston, with respect to the measured foliation defined by the translational part of the holonomy of the spacetime. It is an interesting problem to understand the corresponding picture in the higher dimensional case, see [6] for results in this direction. As discussed below, the level sets of the cosmological time function in a flat simplicial spacetime have locally conformally flat induced geometry. Recall that $H^{1}\left(\Gamma, \mathbb{R}_{\text {vec }}^{3+1}\right)=H^{1}\left(\Gamma, \mathfrak{s o}(4,1)_{\text {Ad }}\right)$. This raises the possibility that the cosmological time foliation in a general $3+1$ dimensional flat spacetime gives a parametrization of the identity component of the deformation space of flat conformal structures on $M$, in a way analogous to the $2+1$ dimensional case described above. The situation in dimension 3 is however more complicated than in dimension 2, as shown by examples of Scannell [17], which demonstrate that the moduli space of flat conformal structures can have an infinite number of components, even when the cohomology $H^{1}\left(\Gamma, \mathfrak{s o}(4,1)_{\mathrm{Ad}}\right)$ vanishes. Since the moduli space of MGHF $n+1$ dimensional spacetimes with 
compact hyperbolic Cauchy surface is a manifold [1], if the above mentioned relation holds, it would imply the conjecture of Kapovich [11, Conjecture 2], that the space of flat conformal structures on a compact hyperbolic manifold is smooth at the hyperbolic structure in dimension 3.

\section{CMC hypersurfaces in wedge spacetimes.}

In this section, we will consider the CMC foliations of wedge spacetimes, and show that the the induced geometry of CMC slices converges to an interval as $\tau \rightarrow-\infty$, i.e. in the direction of the singularity.

Let $(M, g)$ be a compact hyperbolic manifold of sectional curvature -1 , with compact totally geodesic embedded hypersurface $\Sigma$, and denote the induced hyperbolic (if $n \geq 3$ ) metric on $\Sigma$ by $h$. Let $V=(0, \infty) \times M$ be the flat Lorentz cone over $M$ with metric

$$
d s^{2}=-d \rho^{2}+\rho^{2} g, \quad \rho \in(0, \infty) .
$$

For $\ell>0$, the wedge spacetime $V_{\ell}$ is $V$, with the cone over $\Sigma$ replaced by the wedge $W_{\ell}$ of width $\ell$, given by

$$
W_{\ell}=(0, \infty) \times \Sigma \times I_{\ell}
$$

with metric

$$
-d \rho^{2}+\rho^{2} h+d r^{2}, \quad(\rho, r) \in(0, \infty) \times I_{\ell} .
$$

$V_{\ell}$ is a MGHF simplicial spacetime which is a deformation of $V$. The above type of deformation was called elementary in [5]. It will be useful to pass to the covering of these spacetimes defined w.r.t. the fundamental group $\pi_{1}(\Sigma)$. We use notation of the form $\tilde{V}_{\ell}$ or $\mathrm{U}_{\Sigma}\left(V_{\ell}\right)$ for this cover, while $U(V)$ denotes the universal cover. Let $I_{+}^{n+1}(\{0\})$ denote the interior of the future light cone of the origin in $\mathbb{M}^{n+1}$. Then $\tilde{W}_{\ell}=I_{+}^{n}(\{0\}) \times I_{\ell}$. In coordinates $t, y, r, \tilde{W}_{\ell}$ is the set $-t^{2}+|y|^{2}<0$, with metric

$$
-d t^{2}+d y^{2}+d r^{2}
$$

The level sets $\tilde{M}_{\rho}$ of $\rho$ in $\tilde{V}_{\ell}$ have metric $\rho^{2} g$ in $(\widetilde{V \backslash \Sigma})$ and metric $\rho^{2} h$ in $\mathrm{U}\left(\Sigma \times I_{\ell}\right)$. This metric is $C^{1}$ but not $C^{2}$, the second derivatives being bounded, but not continuous, and it is conformally flat. To see this explicitly, note that in the Gauss foliation based on $\Sigma$, the metric $g$ can be written in the form

$$
g=\cos ^{-2}(v)\left(d v^{2}+h\right), \quad v \geq 0,
$$


where $v=0$ at $\Sigma$. The metric $g$ has constant sectional curvature and is in particular locally conformally flat, and hence the wedge metric $d v^{2}+h$ is also locally conformally flat.

\subsection{Mean curvature of $M_{\rho}$.}

The second fundamental form is $K=-\frac{1}{2} \partial_{\rho} g(\rho)$. On $M \backslash \Sigma$, we have $K=$ $-\rho^{-1} g$, while on $\Sigma \times I_{\ell}$, we have $K=-\rho^{-1} h \oplus 0$. The mean curvature $\tau=\operatorname{tr} K$ is given by $\tau=-n / \rho$ on $M \backslash \Sigma$ while on $\Sigma \times I_{\ell}, \tau=-(n-1) / \rho$. This means, in particular, that if we choose $\rho_{0}, \rho_{1}$ so that

$$
-\frac{n-1}{\rho_{0}}<\frac{-n}{\rho_{1}}
$$

then

$$
\max \left(\tau||_{M_{\rho_{0}, \ell}}\right)<\min \left(\tau||_{M_{\rho_{1}, \ell}}\right) .
$$

This shows that the level sets $M_{\rho}$ are barriers, in the sense of [3], for the mean curvature equation in $V_{\ell}$, which using the argument of Gerhardt [10] gives an easy proof that the wedge spacetimes $V_{\ell}$ are globally foliated by CMC hypersurfaces. The function $\rho$ defined above is the cosmological time [2] of $V_{\ell}$.

\subsection{CMC hypersurfaces.}

Now consider the CMC hypersurfaces $M_{\tau}$ of mean curvature $\tau<0$, in the unique global CMC foliation of $V_{\ell}$. We will scale $V_{\ell}$ by a factor $\lambda^{2}$, the rescaled metric is $g^{\prime}=\lambda^{2} g$. This has the effect of scaling $\tau$ to $\lambda^{-1} \tau$. We shall choose

$$
\lambda=|\tau| /(n-1),
$$

so that the rescaled version of the hypersurface $M_{\tau}$ has mean curvature $-(n-1)$, and consider the limit as $\tau \rightarrow-\infty$, i.e. as $\lambda \rightarrow \infty$.

The scaling changes $V_{\ell}$ to $V_{\lambda \ell}$, in particular, the wedge in $V_{\lambda \ell}$ is $W_{\lambda \ell}$, which after a change of coordinates $\rho^{\prime}=\lambda \rho, r^{\prime}=\lambda r$, has metric of the form

$$
-\left(d \rho^{\prime}\right)^{2}+\rho^{\prime 2} h_{i j} d x^{i} d x^{j}+d r^{\prime 2}, \quad r^{\prime} \in I_{\ell} .
$$

where $x^{i}, i=1, \ldots, n-1$ is a coordinate system on $\Sigma$. On $\tilde{W}_{\lambda \ell}$, we also have the scaled Minkowski coordinate system $\left(t^{\prime}, y^{\prime}, r^{\prime}\right)=\lambda(t, y, r)$, with metric

$$
-\left(d t^{\prime}\right)^{2}+\left(d y^{\prime}\right)^{2}+\left(d r^{\prime}\right)^{2},
$$


so that $\rho^{\prime 2}=t^{\prime 2}-\left|y^{\prime}\right|^{2}$. We see from this that the scaling has the effect of stretching the wedge $W_{\ell}$ to the wedge $W_{\lambda \ell}$ of width $\lambda \ell$. We denote the unique CMC hypersurface in $V_{\lambda \ell}$ with mean curvature $-(n-1)$ by $M_{\lambda}$. Let $u_{\tau}$ and $u_{\lambda}$ denote the height functions of $M_{\tau}$ and $M_{\lambda}$ with respect to the time function $\rho$, defined by $u_{\tau}=\left.\rho\right|_{M_{\tau}}$ and $u_{\lambda}=\left.\rho^{\prime}\right|_{M_{\lambda}}$ and let $\tilde{u}_{\tau}, \tilde{u}_{\lambda}$ denote the corresponding lifts. Similarly, let $v_{\tau}=\left.t\right|_{\tilde{M}_{\tau}}$ and $v_{\lambda}=\left.t^{\prime}\right|_{\tilde{M}_{\lambda}}$.

In view of the mean curvature of the level sets of $\rho$, we have by the maximum principle, $\lambda^{-1} \leq u_{\tau} \leq \lambda^{-1} n /(n-1)$, and $1 \leq u_{\lambda} \leq n /(n-1)$. The mean curvature of $M_{\lambda}$ is $-(n-1)$, and hence the derivative bounds for constant mean curvature hypersurfaces $[7,19]$ apply to $v_{\lambda}$. It follows that there is a subsequence of $u_{\lambda}$ which converges uniformly in $C^{3}$ on compacts to a hypersurface $M_{\infty}$ with mean curvature $-(n-1)$ in $W_{\infty}$, where $W_{\infty}$ is the Kasner type spacetime $(0, \infty) \times \Sigma \times \mathbb{R}$ with metric

$$
-d \rho^{2}+\rho^{2} h+d r^{2}, \quad-\infty<r<\infty
$$

This spacetime is the product of the flat Lorentz cone over $\Sigma$ with a line.

The conclusion, so far, is that the limiting hypersurface is an entire CMC hypersurface in $W_{\infty}$, with mean curvature $-(n-1)$. Further, due to the fact that $1 \leq u_{\lambda} \leq n /(n-1), M_{\infty}$ lies between the barriers $\rho=1$ and $\rho=n /(n-1)$. In fact, as we will now prove, a surface $M_{\infty}$ with these properties splits as a product. We state this as the following:

Claim 2.1. Let $M$ be an entire CMC hypersurface of mean curvature $-(n-$ 1) in $W_{\infty}$, bounded from above and below by the barriers $N_{1}, N_{2}$

$$
\begin{aligned}
& N_{1}=\left\{\rho=\rho_{1}\right\}, \rho_{1} \leq 1 \\
& N_{2}=\left\{\rho=\rho_{2}\right\}, \rho_{2} \geq n /(n-1)
\end{aligned}
$$

Then, $M$ splits as $M=\Sigma \times \mathbb{R}$ and $M$ coincides with the level set $\rho=1$.

We will prove the claim as a special case of a more general splitting theorem.

Theorem 2.2. Let $W=(0, \infty) \times \Sigma^{n-1} \times \mathbb{R}^{k}$, with metric

$$
d s^{2}=-d \rho^{2}+\rho^{2} h+\left(d z^{1}\right)^{2}+\cdots\left(d z^{k}\right)^{2} .
$$

Let $M$ be an entire CMC hypersurface in $W$ with mean curvature $-(n-1)$, bounded between the barrier surfaces

$$
\begin{array}{ll}
N_{1}=\left\{\rho=\rho_{1}\right\}, & \rho_{1} \leq 1, \\
N_{2}=\left\{\rho=\rho_{2}\right\}, & \rho_{2} \geq n /(n-1) .
\end{array}
$$


Then $M$ splits as a product $M=\Sigma \times \mathbb{R}^{k}$ with metric

$$
h+\left(d z^{1}\right)^{2}+\cdots\left(d z^{k}\right)^{2}
$$

and $M$ coincides with the level set $\rho=1$.

Proof. Recall that the universal cover of the Lorentz cone over $\Sigma$ is $I_{+}^{n}(\{0\})$, the interior of the future light cone in the $n$ dimensional Minkowski space $\mathbb{M}^{n}$. The universal cover $p: \tilde{\Sigma} \rightarrow \Sigma$ induces the universal cover $p: \tilde{W} \rightarrow W$, with $\tilde{W}=I_{+}^{n}(\{0\}) \times \mathbb{R}^{k} \subset \mathbb{M}^{n+k}$, the $n+k$ dimensional Minkowski space. Let $\tilde{M}$ be the lift of $M$ to $\tilde{W}$. Then $\tilde{M}$ is an entire CMC hypersurface in $\tilde{W}$ which we therefore may think of as a CMC hypersurface in $\mathbb{M}^{n+k}$. Introduce coordinates $\left(t, y^{1}, \ldots, y^{n-1}, z^{1}, \ldots, z^{k}\right)$ on $\mathbb{M}^{n+k}$. We will use the notation $x=(y, z)$. Let $|y|^{2}=\left(y^{1}\right)^{2}+\cdots+\left(y^{n-1}\right)^{2},|z|^{2}=\left(z^{1}\right)^{2}+\cdots+\left(z^{k}\right)^{2}$, and define the function $\tilde{\rho}$ on $\mathbb{M}^{n+k}$ by

$$
\tilde{\rho}^{2}=t^{2}-|y|^{2}
$$

Then $\tilde{N}_{i}=\left\{\tilde{\rho}=\rho_{i}\right\}, i=1,2$ are the universal covers of $N_{1}, N_{2}$, and from the maximum principle and the assumptions of the theorem, it follows that $\tilde{M}$ is bounded between $\tilde{N}_{1}$ and $\tilde{N}_{2}$.

We will now make use of some results of Choi and Treibergs [8]. The conclusion of $[8$, Section 4] can be summarized as follows. Let $v$ be the height function of a $\tau \neq 0$ CMC hypersurface $\tilde{M} \subset \mathbb{M}^{n+k}, v=\left.t\right|_{\tilde{M}}$. Let $V_{v}$ be the positive homogenous of degree one function defined by

$$
V_{v}=\lim _{r \rightarrow \infty, r>0} \frac{v(r x)}{r} .
$$

By $\left[8\right.$, Lemma 4.6], the tangent cone to $V_{v}$ at $0, \chi_{V_{v}}$ is given by

$$
\chi_{V_{v}}(0)=\operatorname{conv}\left(L_{v}\right)
$$

the convex hull of some closed subset $L_{v}$ in $\mathbb{H}^{n-1+k}(\infty)$. Here, $\mathbb{H}^{n-1+k}$ may be identified with the unit ball in $\mathbb{R}^{n-1+k}$ with coordinates $(y, z)$, so that $\mathbb{H}^{n-1+k}(\infty) \cong S^{n+k-2}$. Let $E^{n-1}=\left\{(y, z) \in \mathbb{R}^{n-1+k}: z=0\right\}$. By [8, Lemma 4.3], cf. proof of [8, Lemma 4.6]

$$
V_{v}=\sup _{\xi \in L_{v}} x \cdot \xi
$$

We now make the following 
Claim 2.3. $L_{v} \subset S^{n+k-2} \cap E^{n-1}$.

If this holds, then the splitting theorem [8, Theorem 4.8] shows that in fact $\tilde{M}$ splits as $\tilde{M}^{\prime n-1} \times \mathbb{R}^{k}$. The claim will follow if $V_{v}(0, z)=0$ for all $z \in \mathbb{R}^{k}$. Let $v_{2}$ be the height function of the future barrier $N_{2}$. By construction, $v_{2}(y, z)=w_{2}(y)$, in particular $w_{2}$ is independent of $z$. It follows that $V_{v_{2}}(0, z)=0$. Since $v(x) \leq v_{2}(x)$, we have $V_{v}(0, z) \leq V_{v_{2}}(0, z)$ and hence $V_{v}(0, z)=0$, which proves the claim. It follows that $L_{v} \subset S^{n+k-2} \cap E^{n-1}$ and hence $\tilde{M}$ splits as a metric product $\tilde{M}=\tilde{M}^{\prime n-1} \times \mathbb{R}^{k}$, where $\tilde{M}^{\prime n-1}$ is a CMC hypersurface of $\mathbb{M}^{n}$.

Applying this result to the universal cover $\tilde{M}$ of $M$ as described above, we see that the splitting also applies to $M$ and the conclusion is that $M$ splits as $M=\Sigma^{n-1} \times \mathbb{R}^{k}$. By assumption, the mean curvature of $M$ is $-(n-1)$ which due to the split of $M$ implies that $M=\{\rho=1\}$.

Going back to the limiting process, we see that we have proved that $M_{\lambda}$ converges on compacts to the metric product $\Sigma \times \mathbb{R}$. In terms of the height function $u_{\lambda}$, we have proved

Lemma 2.4. $u_{\lambda}$ converges uniformly in $C^{2}$ on compacts in $W_{\lambda \ell}$ to the constant function 1 .

By the barrier construction, we have

$$
1<u_{\lambda}<\frac{n}{n-1}
$$

or

$$
\lambda^{-1}<u_{\tau}<\lambda^{-1} \frac{n}{n-1} .
$$

The second fundamental form $K$ of $M_{\tau}$ satisfies $K \leq 0$ with our conventions. This means that the height functions w.r.t. $t$ and $t^{\prime}, v_{\tau}$ and $v_{\lambda}$ are convex, cf. [8, Prop. 1.1], in particular

$$
\partial_{r}^{2} v_{\tau}(y, r) \geq 0
$$

We have

$$
v_{\tau}^{2}=\tilde{u}_{\tau}^{2}+|y|^{2}
$$

From the above, $\tilde{u}_{\tau}$ varies by at most $\lambda^{-1}$ which means that $\mid v_{\tau}\left(y, r_{1}\right)-$ $v_{\tau}\left(y, r_{2}\right) \mid \leq \frac{1}{(n-1) \lambda}$. Further, if we restrict to one fundamental domain of $\tilde{M}_{\tau}$, the projection on the $y$-variables is bounded by $C \lambda^{-1}$.

We shall need the following elementary calculus Lemma. 
Lemma 2.5. Let $f:[a, b] \rightarrow \mathbb{R}$ be a convex $C^{2}$ function which takes values in $[0, \Delta]$. Then for any $\epsilon>0, \epsilon<(b-a) / 2$, the estimate

$$
\left|f^{\prime}(x)\right| \leq \frac{\Delta}{\epsilon}
$$

holds in the interval $[a+\epsilon, b-\epsilon]$.

Let $I_{\ell, \epsilon}=(\epsilon, \ell-\epsilon)$. Note that $u_{\tau} \partial_{r} u_{\tau}=v_{\tau} \partial_{r} v_{\tau}$, and hence in view of the above mentioned bound on $y$ in a fundamental domain of $\tilde{M}_{\tau}$, and the lower bound on $u_{\tau}$, the Lemma applies to $\partial_{r} u_{\tau}$ to give an estimate of the form

$$
\left|\partial_{r} u_{\tau}\right| \leq \frac{C}{\lambda \epsilon}, \quad \text { for } r \in I_{\ell, \epsilon} .
$$

The derivative bounds give $\left|D^{\prime} v_{\lambda}\right| \leq C,\left|D^{\prime 2} v_{\lambda}\right| \leq C,\left|D^{\prime 3} v_{\lambda}\right| \leq C$ over compacts. Taking into account the boundedness of the fundamental domain of $\pi_{1}(\Sigma)$ in $\tilde{\Sigma}$ and consequently in $M_{\lambda}$, and the relation of $u_{\lambda}$ to $v_{\lambda}$, we have the corresponding bounds for $u_{\lambda}$. The same bounds hold also in terms of the coordinates $x, r^{\prime}, r=\lambda r^{\prime}$ on $\Sigma \times \lambda I_{\ell}$.

Now, we consider $u_{\tau}$, and note that this is just a rescaling of $u_{\lambda}$ by a factor $1 / \lambda$,

$$
u_{\tau}\left(x, r^{\prime}\right)=\lambda^{-1} u_{\lambda}\left(x, r^{\prime}\right) .
$$

This gives, in view of the fact that the $x$-coordinate does not scale,

$$
\left|D_{x}^{k} u_{\tau}\right| \leq C / \lambda, \quad k=1,2,3 .
$$

From Lemma 2.4, we have also $\left|D_{r} u_{\tau}\right| \leq \frac{C}{\lambda \epsilon}$, for $r \in I_{\ell, \epsilon}$. Without the use of the Lemma, we would just have an estimate of the form $\left|D_{r} u_{\tau}\right| \leq C$.

Lemma 2.6. Fix $\left(x_{0}, r_{0}\right) \in \Sigma \times I_{\ell}$. Then

$$
\lim _{\lambda \rightarrow \infty} \lambda D_{x} u_{\tau}\left(x_{0}, r_{0}\right) \rightarrow 0 .
$$

Proof. We have $\lambda D_{x} u_{\tau}(x, r)=D_{x} u_{\lambda}\left(x, r^{\prime}\right)$. Let $r_{0}^{\prime}=\lambda r_{0}, \bar{r}=r^{\prime}-r_{0}^{\prime}$ and $\bar{u}_{\lambda}\left(x, r^{\prime}\right)=u_{\lambda}\left(x, r^{\prime}-r_{0}^{\prime}\right)$. This has the effect of translating $r_{0}^{\prime}$ to 0 . The derivative bounds apply to $\bar{u}_{\lambda}$ and hence also the conclusion of Theorem 2.2, which implies that $\bar{u}_{\lambda} \rightarrow 1$ in $C^{2}$ on compacts. The result follows.

To compute the induced metric on $M_{\tau}$, we work in coordinates $(x, r), x=$ $\left(x^{1}, \ldots, x^{n-1}\right)$ on $\Sigma \times I_{\ell}$, and define the map $\Phi_{\tau}: \Sigma \times I_{\ell} \rightarrow W_{\ell}$, by

$$
\Phi_{\tau}(x, r)=\left(u_{\tau}(x, r), x, r\right) .
$$


Then the image of $\Phi_{\tau}$ is precisely $M_{\tau} \cap W_{\ell}$. Let the indices $i, j$ run over $1, \ldots, n-1$ and let the index $n$ correspond to the coordinate $r$. Pulling back the metric $-d \rho^{2}+\rho^{2} h+d r^{2}$ by $\Phi_{\tau}$ gives

$$
g_{\tau}=u_{\tau}^{2} h+d r \otimes d r-d u_{\tau} \otimes d u_{\tau}, \quad \text { in } M_{\tau} \cap W_{\ell},
$$

which shows that $g_{\tau} \leq u_{\tau}^{2} h+d r \otimes d r$ as quadratic forms. From this, it follows that

$$
\operatorname{det} g_{\tau} \leq u_{\tau}^{2(n-1)} \operatorname{det} h, \quad \text { in } M_{\tau} \cap W_{\ell} .
$$

Similarly, we have

$$
\operatorname{det} g_{\tau} \leq u_{\tau}^{2 n} \operatorname{det} g, \quad \text { in } M_{\tau} \backslash W_{\ell},
$$

where $g$ is the hyperbolic metric on $M$. From this, it follows, in particular, that

$$
\lim _{\lambda \rightarrow \infty} \lambda^{n-1} \operatorname{Vol}\left(M_{\tau} \backslash W_{\ell}\right)=0 .
$$

We have in view of the fact that $\lambda u_{\tau} \leq n /(n-1)$,

$$
\lambda^{n-1} \int_{\Sigma \times\left(I_{\ell} \backslash I_{\ell, \epsilon}\right)} \sqrt{\operatorname{det} g_{\tau}} d x d r \leq C \epsilon \operatorname{Vol}(\Sigma) .
$$

First, consider the case $n=2$. Then $\Sigma$ is 1 dimensional with metric $h d x^{2}$, and the explicit form of $\operatorname{det} g_{\tau}$ is

$$
\operatorname{det} g_{\tau}=\left[1-\left(\frac{\partial u_{\tau}}{\partial r}\right)^{2}\right] u_{\tau}^{2} h-\left(\frac{\partial u_{\tau}}{\partial x}\right)^{2} \leq u_{\tau}^{2} h .
$$

Here, we may take $h \equiv 1$ by choosing $x$ to be the arclength parameter on $\Sigma$. By Theorem 2.2, $\lambda u_{\tau} \rightarrow 1$, and by Lemma $2.6 \lambda \partial u_{\tau} / \partial x \rightarrow 0$, pointwise as $\lambda \rightarrow \infty$. The dominated convergence theorem now shows

$$
\lambda \int_{\Sigma \times I_{\ell, \epsilon}} \sqrt{\operatorname{det} g_{\tau}} d x d r=(\ell-2 \epsilon) L(\Sigma),
$$

where $L(\Sigma)$ denotes the length of $\Sigma$. Since $\epsilon>0$ is arbitrary, we conclude

$$
\lim _{\lambda \rightarrow \infty} \lambda \operatorname{Vol}\left(M_{\tau} \cap W_{\ell}\right)=\ell L(\Sigma)
$$

Finally, by (2.7), we have

$$
\lim _{\lambda \rightarrow \infty} \lambda \operatorname{Vol}\left(M_{\tau}\right)=\ell L(\Sigma) .
$$


For $n \geq 3$, working in an $h$-orthonormal frame with $e_{n-1}$ proportional to $D_{x} u_{\tau}$, so that $D_{x} u_{\tau}=u_{\tau, x} e_{n-1}$, with $\left|D_{x} u_{\tau}\right|_{h}^{2}=u_{\tau, x}^{2}$, we have

$$
g_{\tau}=\left(\begin{array}{ccc}
u_{\tau}^{2} h_{\perp} & 0 & 0 \\
0 & -u_{\tau, x}^{2}+u_{\tau}^{2} h_{/ /} & -u_{\tau, x} \partial_{r} u_{\tau} \\
0 & -u_{\tau, x} \partial_{r} u_{\tau} & -\left(\partial_{r} u_{\tau}\right)^{2}+1
\end{array}\right),
$$

where $h_{\perp}, h_{/ /}$the restriction of $h$ to $e_{n-1}^{\perp}$ and to $e_{n-1}$ respectively. This gives

$$
\operatorname{det} g_{\tau}=\operatorname{det}\left(u_{\tau}^{2} h_{\perp}\right)\left(\left(1-\left(\partial_{r} u_{\tau}\right)^{2}\right) u_{\tau}^{2} h_{/ /}-\left|D_{x} u_{\tau}\right|_{h}^{2}\right) \text {. }
$$

Taking into account $\operatorname{det} h=\left(\operatorname{det} h_{\perp}\right) h_{/ /}$, and arguing by analogy with the case $n=2$ shows that

$$
\lim _{\lambda \rightarrow \infty} \lambda^{n-1} \operatorname{Vol}\left(M_{\tau}\right)=\ell \operatorname{Vol}(\Sigma)
$$

Next, we consider the distance function on $M_{\tau}$. Let $p, q \in M_{\tau} \cap W_{\ell}$ and let $\gamma$ be a curve connecting $p, q$. We may restrict our consideration to curves such that $d r(\dot{\gamma}) \neq 0$. By parametrizing $\gamma=\gamma(s)$ so that $d r(\dot{\gamma})=1$, we have

$$
|\dot{\gamma}|_{g_{\tau}}^{2}=u_{\tau}^{2}\left|\dot{\gamma}_{x}\right|_{h}^{2}+1-\left|d u_{\tau}(\dot{\gamma})\right|^{2}=1+O\left(\lambda^{-2}\right)
$$

and hence

$$
L[\gamma]=|r(p)-r(q)|+O\left(\lambda^{-2}\right) .
$$

We state the conclusions of this section as

Theorem 2.7. 1. $\lim _{\tau \rightarrow-\infty} \lambda^{n-1} \operatorname{Vol}\left(M_{\tau}\right)=\ell \operatorname{Vol}(\Sigma)$,

2. As $\tau \rightarrow-\infty$, the geometry of $M_{\tau}$ converges in the Gromov sense to the interval of length $\ell$.

\section{CMC hypersurfaces in simplicial flat spacetimes.}

Let $(M, g)$ be a compact hyperbolic manifold with metric $g$ of sectional curvature -1 , of dimension $n \geq 2$, and let $\mathcal{L}=\left\{\left(\Sigma_{k}, \ell_{k}\right), k=1, \ldots, m\right\}$ be a weighted, finite collection of non-intersecting, compact, simple, totally geodesic hypersurfaces with weights $\ell_{k} \in \mathbb{R}$, in $(M, g)$. Further, let $V$ be the simplicial flat spacetime obtained by performing elementary deformations w.r.t. the elements $\left(\Sigma_{k}, \ell_{k}\right)$ of $\mathcal{L}$. Let $(\mathcal{T}, d)$ be the simplicial $\mathbb{R}$-tree dual to $\mathcal{L}$.

Let $M_{\tau}$ be the leaves of the global CMC foliation of $V$, and let $\ell_{M_{\tau}}, s_{\mathcal{T}}$ be the marked length spectrum of $M_{\tau}$ and the marked measure spectrum of 
$\mathcal{T}$, respectively. The conclusion of Theorem 2.7 generalizes immediately, cf. the discussion in section 1.1, to the situation of simplicial flat spacetimes.

Theorem 3.1. With the notation introduced above, the following holds.

1. $\lim _{\tau \rightarrow-\infty} \tilde{M}_{\tau}=\mathcal{T}$, where the limit is understood in the Gromov sense. Thus, the induced geometry on the universal cover $\tilde{M}_{\tau}$ converges, as $\tau \rightarrow-\infty$, in the Gromov sense to $(\mathcal{T}, d)$.

2. $\lim _{\tau \rightarrow-\infty} \ell_{M_{\tau}}=s_{\mathcal{T}}$

\section{Dirichlet energy and rescaled Hamiltonian.}

The Gauss map $\varphi: M_{\tau} \rightarrow M$ is harmonic from the CMC hypersurface $M_{\tau}$ with its induced geometry to $M$ with its hyperbolic geometry [1]. Further, $\varphi$ is the unique harmonic map $M_{\tau} \rightarrow M$ isotopic to the identity. The harmonic map (Dirichlet) energy of $\varphi$, defined by $E\left(M_{\tau}, \varphi\right)=\int_{M_{\tau}}|d \varphi|^{2} \mu_{g}$, can be written as

$$
E\left(M_{\tau}, \varphi\right)=\int_{M}|K|^{2} d \mu_{g}=\int_{M_{\tau}} R \mu_{g}+\tau^{2} \operatorname{Vol}(M, g)
$$

In case $n=2, \int_{M} R \mu_{g}=4 \pi \chi(M)$ by Gauss-Bonnet, which gives the interesting formula

$$
E\left(M_{\tau}, \varphi\right)=4 \pi \chi\left(M_{\tau}\right)+\tau^{2} \operatorname{Vol}\left(M_{\tau}, g\right)
$$

found by Puzio [15].

The rescaled Hamiltonian $\mathcal{H}=|\tau|^{n} \operatorname{Vol}\left(M_{\tau}\right)$ is the Hamiltonian for gravity in a suitably chosen gauge, see [9]. As shown in [1], it satisfies

$$
\mathcal{H} \geq n^{n} \operatorname{Vol}(M, g) .
$$

Equality in (4.1) holds if and only if $V$ is the Lorentz cone over $(M, g)$.

We will use our results on flat simplicial spacetimes to understand the limiting behavior of the Dirichlet energy and the rescaled Hamiltonian. Let $V, M, \mathcal{L}$ be as in Section 3, and let $M_{\tau}, M_{\lambda}$ be the leaves of the CMC foliation of $V$ and the rescaled leaves, respectively. If we let $\lambda=|\tau| /(n-1)$ as above, then

$$
E\left(M_{\lambda}, \varphi\right)=\lambda^{n-2} E\left(M_{\tau}, \varphi\right)
$$

is scale invariant. Let $K_{\lambda}$ be the second fundamental form of $M_{\lambda}$. Since we know from the above that for a wedge spacetime, the height functions 
$u_{\lambda} \rightarrow w_{\lambda}$, we are able to conclude in the simplicial case, from the bounds on the derivatives of $u_{\lambda}$ that $K_{\lambda} \rightarrow-h \oplus 0$, on each wedge, where $h$ is the metric on $\Sigma$. We have $|h \oplus 0|^{2}=n-1$, which taking into account the fact that the contribution from the part of $M_{\lambda}$ off the wedge can be ignored by the arguments above, gives that the contribution from each wedge to $E\left(M_{\lambda}, \varphi\right)$ behaves like $(n-1) \operatorname{Vol}\left(M_{\lambda}\right)$. By $(4.2)$, this gives $\lim _{\lambda \rightarrow \infty} \lambda^{n-3} E\left(M_{\tau}, \varphi\right)=$ $(n-1) \sum_{k=1}^{m} \ell_{k} \operatorname{Vol}\left(\Sigma_{k}\right)$.

The rescaled Hamiltonian $\mathcal{H}$ is scale invariant, so we can consider its behavior on $M_{\lambda}$. Here, we have mean curvature approximate to 1 in the wedges, while the wedges have length $\lambda \ell_{k}$, which gives $\lim _{\lambda \rightarrow \infty} \lambda^{-1} \mathcal{H}=$ $\sum_{k=1}^{m} \ell_{k} \operatorname{Vol}\left(\Sigma_{k}\right)$. Summarizing, we have

Theorem 4.1. 1. $\lim _{\lambda \rightarrow \infty} \lambda^{n-3} E\left(M_{\tau}, \varphi\right)=(n-1) \sum_{k=1}^{m} \ell_{k} \operatorname{Vol}\left(\Sigma_{k}\right)$,

2. $\lim _{\lambda \rightarrow \infty} \lambda^{-1} \mathcal{H}=\sum_{k=1}^{m} \ell_{k} \operatorname{Vol}\left(\Sigma_{k}\right)$.

Specializing to the $2+1$ dimensional case, we have denoted the length of $\Sigma$ by $L(\Sigma), \lim _{\lambda \rightarrow \infty} \lambda^{-1} E\left(M_{\tau}, \varphi\right)=\sum_{k=1}^{m} \ell_{k} L\left(\Sigma_{k}\right)$, and similarly for $\mathcal{H}$.

Let us compare this result to what is known about the time dependence of the Dirichlet energy in the $2+1$ dimensional case. It has been proved [4, Lemma 4.4] that with our present conventions,

$$
\begin{aligned}
A\left(M_{\tau_{0}}\right)\left|\tau_{0}\right|^{2} /|\tau| & \leq|\tau| A\left(M_{\tau}\right) \leq\left|\tau_{0}\right| A\left(M_{\tau_{0}}\right), \quad \text { for } \tau<\tau_{0}<0 \\
A\left(M_{\tau_{0}}\right)\left|\tau_{0}\right| & \leq|\tau| A\left(M_{\tau}\right) \leq\left|\tau_{0}\right|^{2} A\left(M_{\tau_{0}}\right) /|\tau|, \quad \text { for } \tau_{0}<\tau<0
\end{aligned}
$$

This together with Puzio's result gives

$$
\left.A\left(M_{\tau_{0}}\right)\left|\tau_{0}\right|^{2} \leq E\left(M_{\tau}, \varphi\right)-4 \pi \chi\left(M_{\tau}\right) \leq A\left(M_{\tau_{0}}\right)\right)|\tau|\left|\tau_{0}\right|, \quad \text { for } \tau<\tau_{0}<0
$$

which gives the correct leading order behavior in the collapsing direction, but which does not identify the coefficient. Similarly, in the expanding direction, we know that

$$
\lim _{\tau \rightarrow 0}|\tau|^{2} A\left(M_{\tau}\right)=4 A(M, g),
$$

where $A(M, g)$ is the area of the hyperbolic geometry on $M$. Therefore, we find that

$$
\lim _{\tau \rightarrow 0} E\left(M_{\tau}, \varphi\right)=4 \pi \chi\left(M_{\tau}\right)+4 A(M, g)=4 \pi|\chi(M)|
$$

\section{Acknowledgements.}

The author is grateful to Vince Moncrief, Ralph Howard, Riccardo Benedetti and Kevin Scannell for helpful discussions on various aspects of this material. 


\section{References.}

[1] Lars Andersson, Constant mean curvature foliations of flat space-times, Comm. Anal. Geom. 10 (5) (2002), 1125-1150.

[2] Lars Andersson, Gregory J. Galloway, and Ralph Howard, The cosmological time function, Classical Quantum Gravity 15 (2) (1998), 309-322.

[3] Lars Andersson, Gregory J. Galloway, and Ralph Howard, A strong maximum principle for weak solutions of quasi-linear elliptic equations with applications to Lorentzian and Riemannian geometry, Comm. Pure Appl. Math. 51 (6) (1998), 581-624.

[4] Lars Andersson, Vincent Moncrief, and Anthony J. Tromba, On the global evolution problem in $2+1$ gravity, J. Geom. Phys. 23 (3-4) (1997), 191-205.

[5] Riccardo Benedetti and Enore Guadagnini, Cosmological time in $(2+1)$ gravity, Nuclear Phys. B 613 (1-2) (2001), 330-352.

[6] Francesco Bonsante, Flat spacetimes with compact hyperbolic Cauchy surfaces, preprint math.DG/0311019, 2003.

[7] Shiu Yuen Cheng and Shing Tung Yau, Maximal space-like hypersurfaces in the Lorentz-Minkowski spaces, Ann. of Math. (2) 104 (3) (1976), 407-419.

[8] Hyeong In Choi and Andrejs Treibergs, Gauss maps of spacelike constant mean curvature hypersurfaces of Minkowski space, J. Differential Geom. 32 (3) (1990), 775-817.

[9] Arthur E. Fischer and Vincent Moncrief, The Einstein flow, the $\sigma$ constant and the geometrization of 3-manifolds, Classical Quantum Gravity 16 (11) (1999), L79-L87.

[10] Claus Gerhardt, H-surfaces in Lorentzian manifolds, Comm. Math. Phys. 89 (4) (1983), 523-553.

[11] Michael Kapovich, Deformations of representations of discrete subgroups of $\mathrm{SO}(3,1)$, Math. Ann. 299 (2) (1994), 341-354.

[12] Michael Kapovich and John J. Millson, On the deformation theory of representations of fundamental groups of compact hyperbolic 3manifolds, Topology 35 (4) (1996), 1085-1106. 
[13] Geoffrey Mess, Lorentz spacetimes of constant curvature, Tech. Report IHES/M/90/28, Institute des Hautes Etudes Scientifiques, 1990.

[14] Jean-Pierre Otal, Le théorème d'hyperbolisation pour les variétés fibrées de dimension 3, Astérisque (1996), no. 235, $\mathrm{x}+159$.

[15] Raymond S. Puzio, The Gauss map and $2+1$ gravity, Classical Quantum Gravity 11 (11) (1994), 2667-2675.

[16] Kevin P. Scannell, Infinitesimal deformations of some $\mathrm{SO}(3,1)$ lattices, Pacific J. Math. 194 (2) (2000), 455-464.

[17] Kevin P. Scannell, Local rigidity of hyperbolic 3-manifolds after Dehn surgery, Duke Math. J. 114 (1) (2002), 1-14.

[18] Richard M. Schoen and Shing Tung Yau, Existence of incompressible minimal surfaces and the topology of three-dimensional manifolds with non-negative scalar curvature, Ann. of Math. (2) 110 (1) (1979), 127142.

[19] Andrejs E. Treibergs, Entire spacelike hypersurfaces of constant mean curvature in Minkowski space, Invent. Math. 66 (1) (1982), 39-56.

[20] Anthony J. Tromba, Teichmüller theory in Riemannian geometry, Lectures in Mathematics ETH Zürich, Birkhäuser Verlag, Basel, 1992, Lecture notes prepared by Jochen Denzler.

[21] Abdelghani Zeghib, Laminations et hypersurfaces géodésiques des variétés hyperboliques, Ann. Sci. École Norm. Sup. (4) 24 (2) (1991), $171-188$.

\section{Department of Mathematics}

UNIVERSITY OF MiAMI

Coral Gables, FL 33124

USA

E-mail address: larsa@math.miami.edu

Received August 8, 2003. 